\title{
Simulation of Laser Structuring of Thin-Film Solar Cells
}

\author{
T. PESCHEL $^{* 1}$, S. NOLTE ${ }^{* 2}$, R. EBERHARDT ${ }^{* 1}$, A. TÜNNERMANN ${ }^{* 1,{ }^{* 2}}$ \\ ${ }^{* 1}$ Fraunhofer-Institute of Applied Optics und Precision Engineering, A.-Eimnstein-Str. 7, \\ 07745 Jena, Germany \\ E-mail: Thomas.peschel@iof.fraunhofer.de \\ ${ }^{* 2}$ Institute of Applied Optics, Friedrich-Schiller-University, A.-Eimnstein-Str. 15, \\ 07745 Jena, Germany
}

\begin{abstract}
We present a theoretical model of the interaction of ultra-short laser pulses with semiconductorbased multilayer structures. In particular the model focuses on laser processing of thin-film solar cells. The propagation of laser light within the thin-film system is described by a finite-difference model in order to determine the distribution of absorbed power. Since light propagation in semiconductors is strongly influenced by screening due to excited carriers we include the dynamics of charge carriers which may be driven either by direct absorption of the laser radiation or by multiphoton absorption and impact ionization of highly excited carriers. Depending on the excitation wavelength and pulse energy, absorption occurs in different depths of the structure which has a large effect on the efficiency of the laser ablation process. In a second step the energy density deposited by the laser beam is transferred to a thermo-mechanical model which describes the actual ablation process. Results from this model are compared with experimental findings.
\end{abstract}

DOI: $10.2961 /$ jlmn.2015.03.0009

Keywords: modelling, laser structuring, short pulses, thin-film solar cells

\section{Introduction}

Photovoltaics are one of the most important technologies for the generation of renewable energy. Because of the large amount of installed and planned systems even small changes in efficiency of the solar cells themselves or in the effort needed for their production may have enormous economic and environmental impact.

Most of the current photovoltaic systems are based on single crystalline silicon. Though this is a mature technology which guarantees for high efficiency, silicon has an indirect band gap, which results in a relatively large absorption length for the incoming light [1]. Thus, relatively thick material of the order of $100 \mu \mathrm{m}$ is required. Furthermore, a considerable amount of material is wasted in the process of wafer cutting. Additional effort is needed for the assembly of individual wafers into complete solar cell modules.

On the other hand, thin-film solar cells rely on direct semiconductors, like e.g. $\mathrm{CuIn}_{1-\mathrm{x}} \mathrm{Ga}_{\mathrm{x}} \mathrm{Se}_{2}$ (CIGS) with an absorption length of some micrometers. Such materials allow to reduce the thickness of the solar cell to well below $10 \mu \mathrm{m}$ and the amount of active material needed to well below $10 \%$ compared to single crystal silicon.

Whole panels may be made on a single glass substrate thus avoiding the effort for the assembly of modules. In order to obtain a usable level of output voltage all cells on one substrate are interconnected in series. These connections are made by cutting trenches (P1 to $\mathrm{P} 3$ as shown in Fig. 1) into the different layers deposited on the substrate.

The area of these interconnects does not contribute to energy generation. Thus, a small width and narrow spacing of the trenches is crucial for a high efficiency of the panel.

Today the P1 scribe is generated by laser ablation while mechanical scribing is used for P2 and P3. The process of mechanical scribing is relatively complex due to the fast degradation of the scribing needles and the necessity for frequent re-adjustment. Mechanical scribing results in relatively wide trenches [2] and requires additional space between the trenches to allow for the crack formation occurring at the edges. Furthermore it is difficult to control the needle position precisely to follow the already existing trenches.

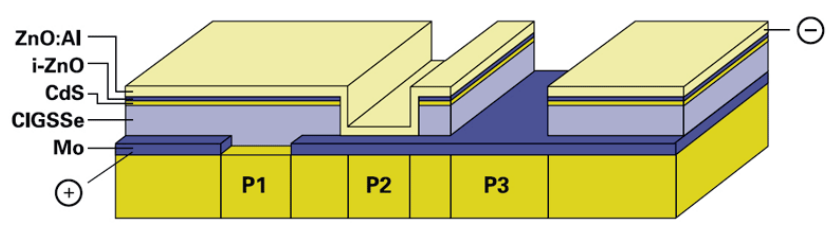

Fig. 1: P1-P3 trenches for the formation of the serial interconnect between two adjacent cells of a CIGS thin-film photovoltaic panel.

Laser structuring allows to reduce the width of the trenches as well as their spacing, which results in an increase of the usable area per cell and, hence, in an increased efficiency. Furthermore, the amount of "wasted" material may be reduced.

In order to reach a high efficiency of the structuring process as well as to reduce thermally induced degradation of the material, we want to deposit as little energy as possible into the solar cell. In particular, it is favorable to use a so-called confined ablation [3-5] where only a small amount of material is molten or vaporized. The remaining material above will blow-off due to gas pressure and thermally induced stress.

An optimum ablation process requires careful tailoring the interaction of the laser beam with the layer system in order to deposit the energy at the optimum depth. 
In the current paper we present a theoretical model which may be used to optimize the ablation process. This model consists of three major components:

1. the description of light propagation in the layer stack of the solar cell,

2. the description of the dynamics of excited carriers and their feedback on light propagation, and

3. a thermo-mechanical model, which describes the actual ablation.

\section{Optical Model}

Thin-film solar cells consist of a stack of different semiconductor materials typically deposited on a glass substrate. A metal layer forms the back contact of the cell. A cut view of a typical layer system is given in fig. 1. Relevant material parameters are summarized in table 1 .

Table 1 Optical properties of the relevant materials.

\begin{tabular}{|c|c|c|c|c|}
\hline $\begin{array}{l}\text { Mate- } \\
\text { rial }\end{array}$ & $\begin{array}{l}\text { Dielectric } \\
\text { const.@ } \\
1064 \mathrm{~nm}\end{array}$ & $\begin{array}{l}\text { Dielectric } \\
\text { const.@ } \\
532 \text { nm }\end{array}$ & $\begin{array}{l}\text { Band } \\
\text { edge } \\
{[\mathrm{nm}]}\end{array}$ & Ref. \\
\hline $\begin{array}{l}\mathrm{TCO} \\
(\mathrm{ZnO}: \\
\mathrm{Al})\end{array}$ & $\begin{array}{c}2.94+ \\
0.03 \mathrm{i}\end{array}$ & $\begin{array}{l}3.78+ \\
0.003 \mathrm{i}\end{array}$ & $(390)$ & $\begin{array}{l}{[5],} \\
{[10]}\end{array}$ \\
\hline $\mathrm{iZnO}$ & 3.72 & 4.16 & 430 & $\begin{array}{l}{[5],} \\
{[10]}\end{array}$ \\
\hline $\mathrm{CdS}$ & 4.67 & $\begin{array}{l}5.76+ \\
0.05 \mathrm{i}\end{array}$ & 530 & {$[1]$} \\
\hline CIGS & $9.3+0.6 \mathrm{i}$ & $\begin{array}{c}10.6+ \\
2.8 \mathrm{i}\end{array}$ & 1200 & {$[5-8]$} \\
\hline MoSe2 & 22.3 & $\begin{array}{l}21.9+ \\
20.8 \mathrm{i}\end{array}$ & 840 & [9] \\
\hline Mo & $\begin{array}{r}-11.9+ \\
20.6 \mathrm{i}\end{array}$ & $\begin{array}{l}1.3+ \\
27.4 \mathrm{i}\end{array}$ & n.a. & {$\left[\begin{array}{lll}1 & 1\end{array}\right]$} \\
\hline
\end{tabular}

The typical thickness of the layer system is of the order of $3 \mu \mathrm{m}$. The respective time of light propagation amounts to $10 \mathrm{fs}$, which is by orders of magnitude faster than the duration of 1 to 10 ps of typical laser pulses used for ablation. Thus, light propagation in the layer system may be considered quasi-static. All dynamics of the interaction is due to the excitation of carriers.

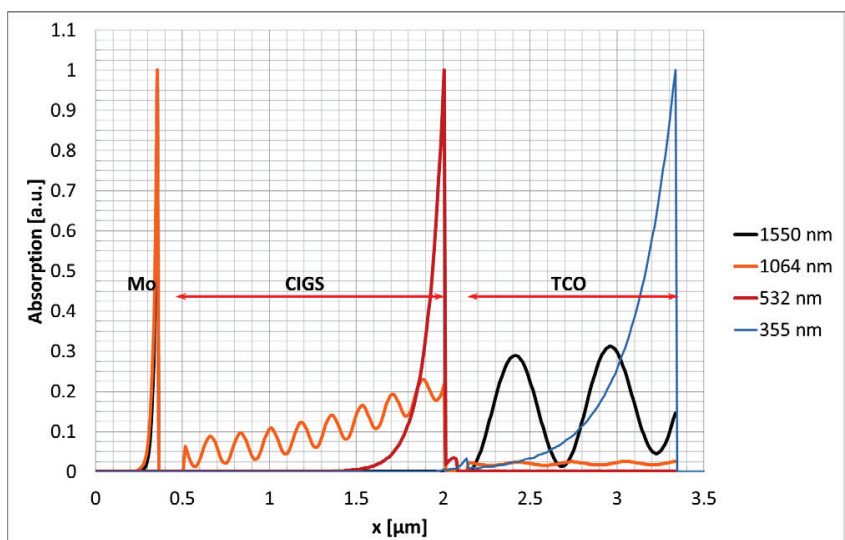

Fig. 2: Vertical distribution of the absorbed laser power for different excitation wavelengths.
To get a first insight in the behavior of the optical system we used a matrix-formalism [13] to describe the linear plane-wave response of the stack. The model takes into account the measured dielectric properties of the individual materials [6-12].

As a result we obtain the total linear absorption as well as the vertical distribution of deposited laser energy over the layer structure (see fig 2).

\subsection{Wavelength dependence}

Depending on the excitation wavelength the dominant absorption occurs in different layers. The resulting differences in the distribution of the energy deposition have a large effect on the efficiency of the respective laser ablation process.

Fig. 2 shows typical energy deposition patterns for the infrared (IR: $1550 \mathrm{~nm}$ ), near infrared (NIR: 1064), visual (VIS: $532 \mathrm{~nm}$ ), and the ultraviolet (UV: $366 \mathrm{~nm}$ ) spectral ranges.

In the in IR range all layers of the thin-film solar cell except the back contact are at least partially transparent. Thus, a large percentage of the laser energy is absorbed at the interface to the underlying Molybdenum film. However, at longer wavelengths, free carriers in the TCO cause increasing absorption.

In the NIR range below $1100 \mathrm{~nm}$ absorption in the CIGS layer sets on. This defines a window in the range of 1100-1200 nm, where it seems possible to deposit most of the energy below the CIGS layer to lift-off the whole stack with relatively low energetic effort.

In the VIS and NIR range absorption takes place predominantly at the top of the CIGS layer. The absorption length increases with wavelength. Energy deposition in the top region of the CIGS as well as in the CdS buffer may lead to a lift-off of the TCO front contact.

In the UV range absorption takes place in the TCO right at the top of the stack. The stack would be ablated layer by layer from its top side down to the back contact.

\subsection{Carrier Dynamics}

The distribution of excited carriers inside the layers results in a corresponding inhomogeneous distribution of induced changes of the refractive index. Thus, we need a three dimensional model of the optical propagation in the layer stack.

If the diameter of the laser beam is large compared to the wavelength, polarization effects will play a minor role. In the following we will restrict ourselves to this case and use the Helmholtz wave equation. The wave equation is discretized according to a finite-difference scheme. Cylindrical symmetry is assumed.

The whole dynamics of the system is attributed to the evolution of the carrier density, which is driven either by direct absorption of the laser radiation or by multi-photon absorption and impact ionization of highly excited carriers [14], depending on the wavelength of excitation. The order of the multiphoton processes is defined by the number $\mathrm{k}$ of photons needed to overcome the band gap of the respective material.

We describe the evolution of the carrier density by a rate equation: 
$\frac{d n_{\text {total }}(t)}{d t}=\frac{\alpha}{k E_{p}} I^{k}(t)+\frac{\eta}{E_{p}} \sigma\left(n_{\text {total }}\right) I(t) n_{\text {hot }}(t)-\frac{n_{\text {total }}(t)}{\tau_{1}}$

The total carrier density $n_{\text {total }}$ is driven by the light intensity $I(t)$. The individual terms on the right-hand side denote multi-photon absorption with the order $k$ (including single photon absorption in the case of $k=1$ ) and a characteristic strength $\alpha$, impact ionization with an efficiency $\eta$ and an absorption cross section $\sigma$, and carrier recombination with the life time $\tau_{1}$. The photon energy is denoted $E_{p}$.

Impact ionization depends on the number of "hot" carriers, the energy of which is larger than $2 E_{\text {gap }}-E_{p}$ [14].

In the case of single photon absorption all excited carriers will contribute to impact ionization, i.e. $n_{\text {hot }}=n_{\text {total }}$. Thus, eq. (1) is already sufficient to describe the carrier dynamics in the single photon case.

In the case of multi-photon excitation the evolution of hot carriers is simulated by a second rate equation for the mean energy of the excited carriers.

$$
\begin{aligned}
\frac{d E(t)}{d t}=\alpha I^{k}(t) & +\sigma\left(n_{\text {total }}\right) I(t) n_{\text {total }}(t) \\
& -\frac{E_{\text {gap }} n_{\text {total }}(t)}{\tau_{1}}-\frac{E_{\text {exc }}}{\tau_{2}}
\end{aligned}
$$

where the excess energy is defined as $E_{\text {exc }}=E(t)-E_{\text {gap }}$. The relaxation of the excess energy is described by the parameter $\tau_{2}$. Actually the excess energy per carrier is equivalent to the carrier temperature in conventional twotemperature models [5].

In order to get a closed model we need an additional relation between the density of hot carriers and the carrier energy. If we consider the stationary limit of Rethfeld's model [14] we find an exponential relation between the number of carriers in a certain level of excitation and the energy of the respective level. Summing up over the distribution we may derive an implicit form of the desired relation (see fig. 3):

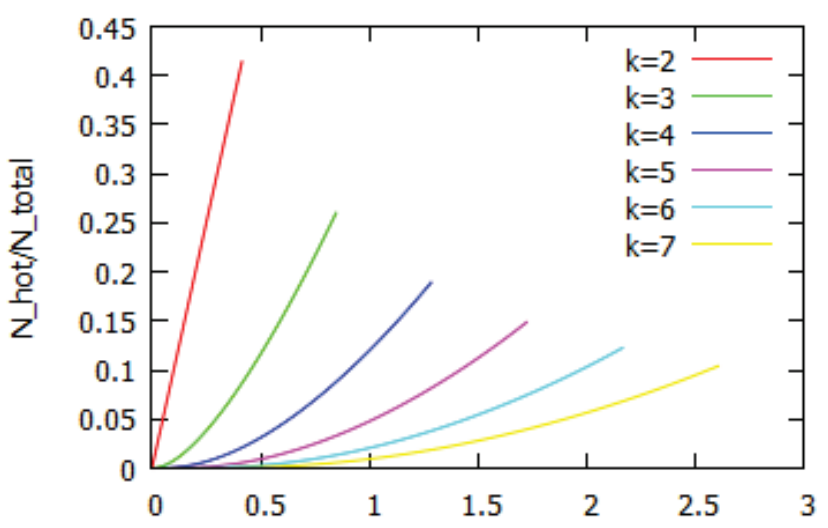

Excess energy per carrier/Photon energy

Fig. 3: Ratio of hot carriers as a function of the excess energy.

The above relation may be approximated well in explicit form by a power-law for each order $k$ :

$$
n_{\text {hot }}(t)=\left(E_{\text {exc }}(t) /\left(E_{p} n_{\text {total }}(t)\right)\right)^{c(k)} n_{\text {total }}(t)
$$

Where the respective exponents for different orders of the process are given in table 2 :
Table 2 Exponents for the determination of the number of hot carriers.

\begin{tabular}{cc}
\hline$k$ & Exponent $c(k)$ \\
\hline 2 & 1 \\
\hline 3 & 1.535 \\
\hline 4 & 1.866 \\
\hline 5 & 2.092 \\
\hline 6 & 2.255 \\
\hline 7 & 2.379 \\
\hline
\end{tabular}

Impact ionization can be started by the initial density of free carriers, which is either the intrinsic one or the one due to doping. Alternatively, free carriers may be created via multi-photon absorption.

The efficiency of multi-photon impact ionization decreases strongly with the number of photons needed since the amount of "hot" carriers decreases (compare fig. 3).

Additionally the efficiency decreases with longer pulse duration, typically in the lower ns range, because the carrier energy will relax significantly during the pulse: Long pulses with photon energies below the band gap of CIGS may penetrate the layer stack without generating a large number of additional free carriers. However, in the case of picosecond pulses the initial carrier density in the CIGS will always be sufficient to start carrier excitation due to impact ionization.

\subsection{Impact of the carrier dynamics on the optical model}

During laser excitation charge carriers accumulate in the regions of light absorption. They cause a modification of the optical properties of the materials via plasma screening [15]:

$$
\chi_{\text {plasma }}(\omega)=\frac{\varepsilon \omega_{p}^{2}}{\omega_{p}^{2}-\omega^{2}-\frac{i e \omega}{\mu m_{e f f}}}
$$

where the plasma frequency $\omega_{\mathrm{p}}$

$$
\omega_{p}^{2}=\frac{e^{2} n_{\text {total }}(t)}{\varepsilon_{0} \varepsilon m_{\text {eff }}}
$$

is driven by the total carrier density $n_{\text {total }}$. The relevant material properties are the dielectric constant $\varepsilon$ (see tab. 1), carrier mobility $\mu$, charge $e$ and effective mass $m_{\text {eff }}[16]$. The plasma susceptibility for CIGS at $1064 \mathrm{~nm}$ is plotted in fig. 4 as a function of the carrier density. The image shows the typical behavior: At lower carrier density the real part of the susceptibility decreases with density. Finally the real part of the dielectric constant turns negative. This resembles the behavior of metals, thus preventing light from penetrating into the respective region.

Additionally, absorption increases dramatically when the carrier density approaches some critical value, which is approximately $9 \times 10^{26} / \mathrm{m}^{3}$ in the current example. 


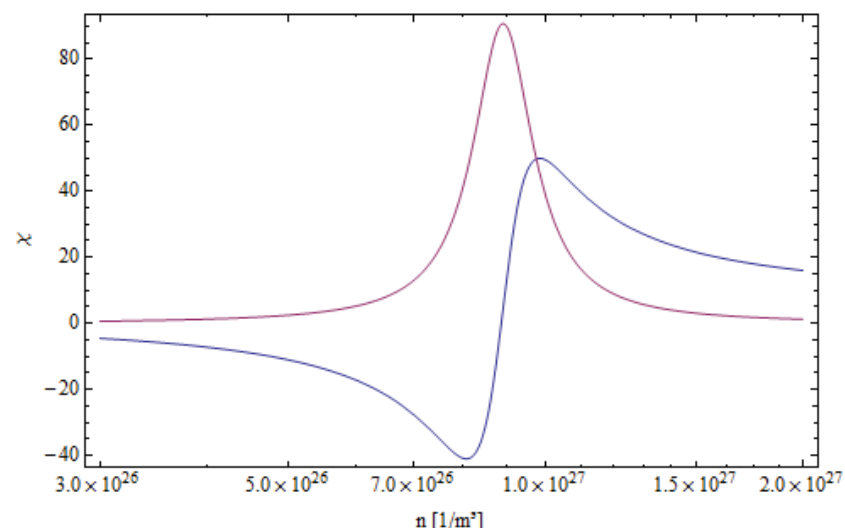

Fig. 4: Plasma susceptibility $\chi$ plasma as a function of the density of free carriers $\mathrm{n}$ for CIGS at $1064 \mathrm{~nm}$. The real part is plotted in blue, the imaginary part in purple. Parameters are taken from $[7,16]$.

\subsection{Results of the optical simulation}

We simulated the interaction of the laser beam with the complete layer stack of a CIGS-based thin film solar cell. This situation corresponds to a P3-structuring. We concentrate on two cases (compare fig. 2):

1. laser excitation with $0.1 \mu \mathrm{J}$ at $1064 \mathrm{~nm}$, where absorption in the Molybdenum layer dominates in the low power limit, and

2. excitation with $1 \mu \mathrm{J}$ at $515 \mathrm{~nm}$, where the maximum absorption occurs at the top of the CIGS layer.

The laser has a beam diameter of $28 \mu \mathrm{m}$ and a pulse duration of $10 \mathrm{ps}$. The initial condition for the charge carrier density was deduced from the ellipsometrically measured dielectric constants of the materials (see Table 1).

In both cases excitation in the CIGS layer is above the band gap, i.e. we have linear absorption $(k=1)$. For excitation at $515 \mathrm{~nm}$ we also have taken into account absorption and the related carrier dynamics in the $\mathrm{CdS}$ buffer layer. Linear absorption by free carriers is taken into account in the heavily doped TCO layer.

The simulations predict a significant change in the sample reflectivity as deposited energy increases. It increases at low energies, while saturation occurs at higher energy. Due to the frequency dependence of plasma shielding (see eq. 4) saturation starts at higher energies, as the wavelength decreases. The results are plotted in figs. 5-6.

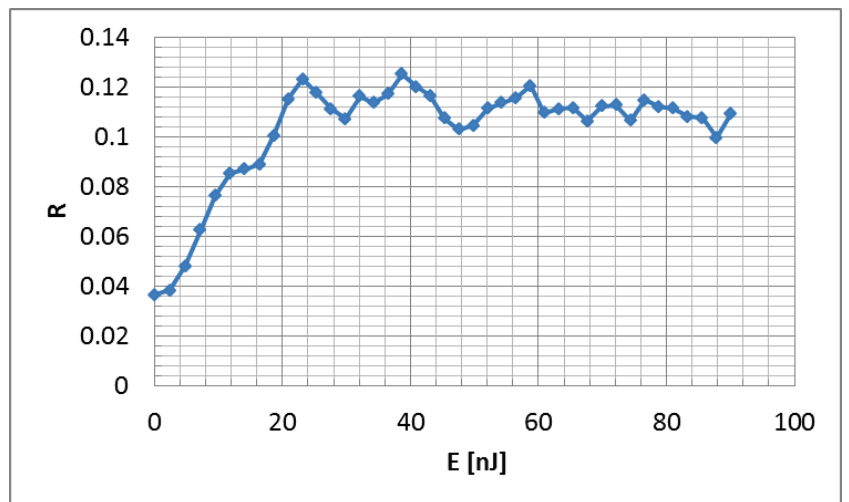

Fig. 5: Reflectivity of the layer system as a function of the deposited energy. The laser wavelength is $1064 \mathrm{~nm}$, the beam diameter $28 \mu \mathrm{m}$.

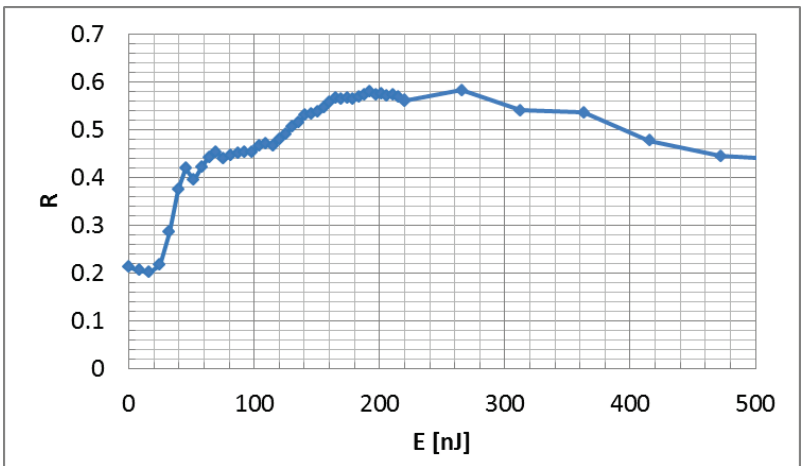

Fig. 6: Reflectivity of the layer system as a function of the deposited energy. The laser wavelength is $515 \mathrm{~nm}$, the beam diameter $28 \mu \mathrm{m}$.
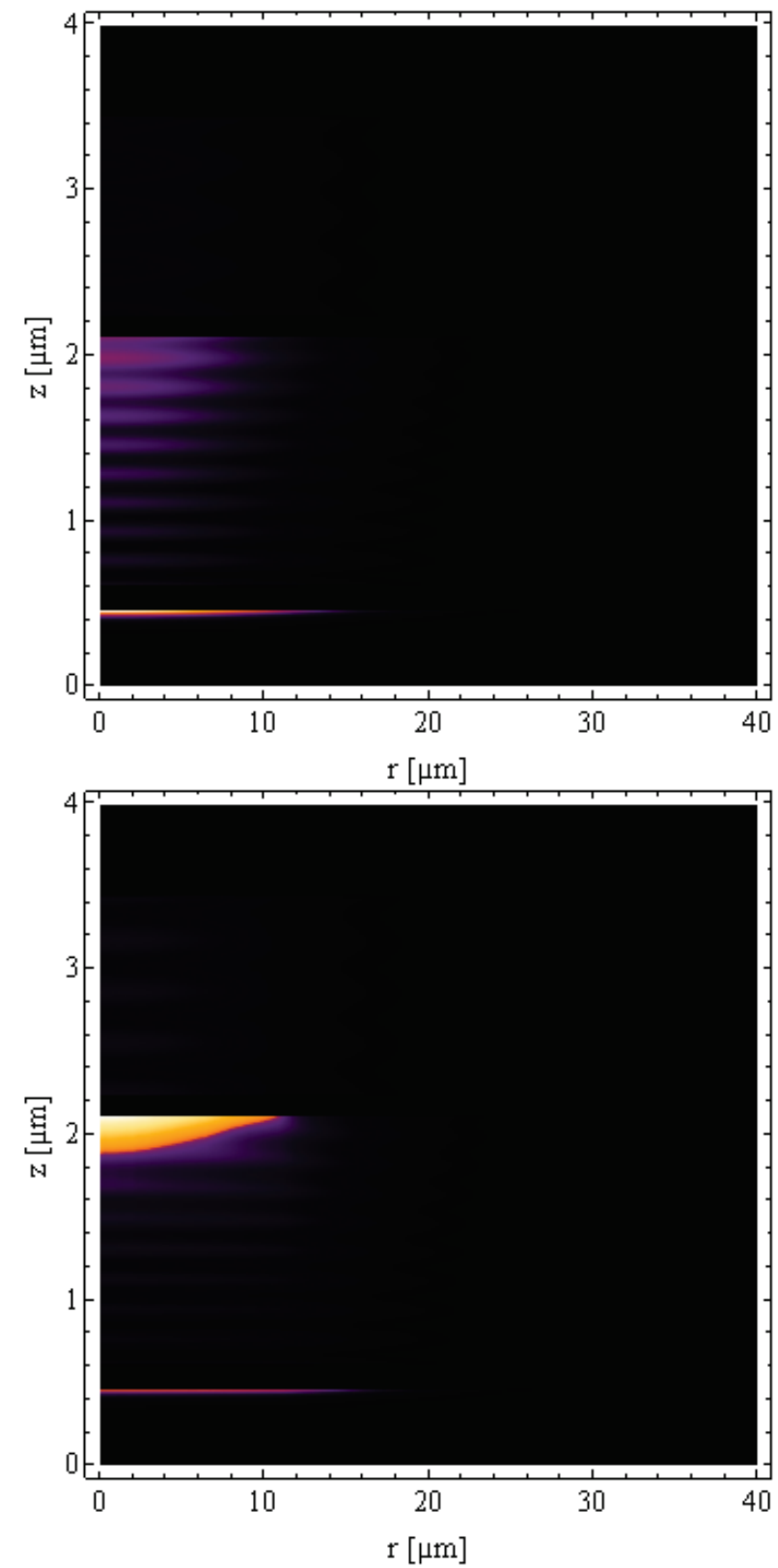

Fig. 7: Distribution of absorbed energy in the layer system for a laser wavelength of $1064 \mathrm{~nm}$. The upper graph shows the initial situation with a strong absorption in the Mo layer. The lower picture shows the distribution after half of the pulse i.e. for a deposited energy of $45 \mathrm{~nJ}$. The pulse duration is $10 \mathrm{ps}$ and the beam diameter is $28 \mu \mathrm{m}$. 

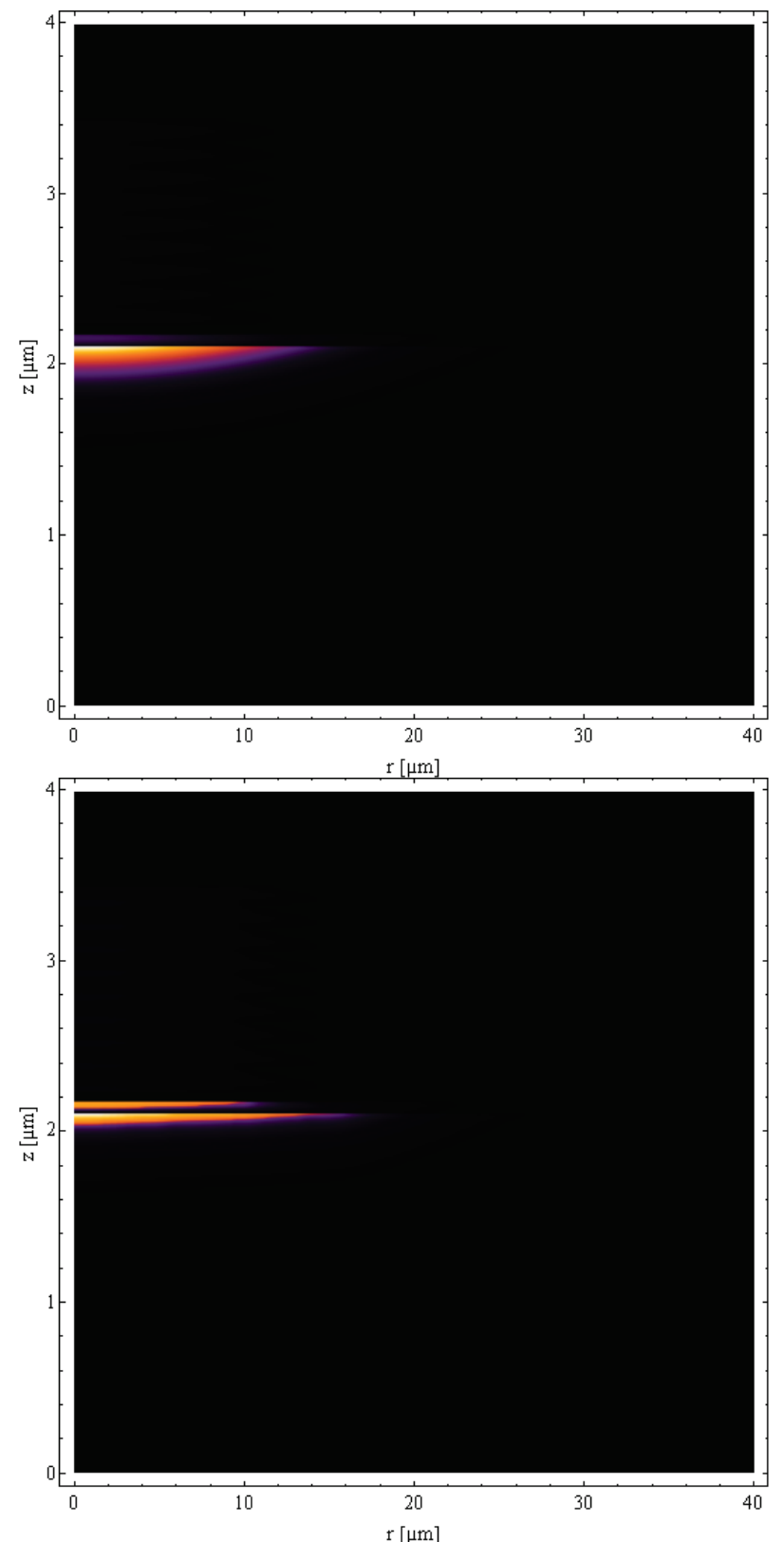

Fig. 8: Distribution of absorbed energy in the layer system for a laser wavelength of $515 \mathrm{~nm}$. The upper graph shows the initial situation at low energy, where most energy is absorbed in the top region of the CIGS layer. The lower picture shows the distribution after $40 \%$ of the pulse i.e. for a deposited energy of $220 \mathrm{~nJ}$. The pulse duration is $10 \mathrm{ps}$ and the beam diameter is $28 \mu \mathrm{m}$.

The effect of plasma shielding is particularly obvious in the case of the excitation at $1064 \mathrm{~nm}$ : The maximum of absorbed energy shifts from the top of the Mo layer in the case of low energy to the top of the CIGS layer. The respective energy distributions are given in fig. 7. As a consequence confined ablation of the whole stack for the P3 scribe is difficult to achieve with this wavelength.

A similar shift occurs for an excitation at $515 \mathrm{~nm}$, though the changes are much smaller. The absorbing region at the top of the CIGS layer shrinks from $90 \mathrm{~nm}$ to $50 \mathrm{~nm}$. Additionally, the absorption in the CdS buffer layer increases (see fig. 8).

As a general rule, shielding will force the absorbing region to shift backwards, i.e. towards the laser source.

\section{Thermo-mechanical model}

In order to characterize the actual ablation behavior and the size of heat-affected zones we use a finite-element based thermo-mechanical model. For the finite-element simulation we used a commercial code. Details on the underlying models are given in [20]. Material properties were taken from the literature $[1,6,11,12]$.

\subsection{Simulation of the P1 scribe}

To get a better understanding of the ablation process, we begin with simulations of the P1 scribe. Material parameters for Molybdenum are readily available from literature [12].

Furthermore, we have the possibility to compare the simulations with experimental results on the ablation of Molybdenum layers [17, 18]. When the laser beam was applied through the glass substrate, the experiments showed confined ablation of complete Mo disks. At high laser fluences holes with traces of molten metal will develop in the centers of the disks.

These results have been compared to finite element simulations of a corresponding transient thermal model. The experimentally observed diameters of the disks correlate well with the diameter of molten glass in the simulation (see fig. 9), which indicates that the ablation diameter is determined by the delamination of the metal from the glass. The diameter of the holes corresponds to the diameter of molten Molybdenum at the top side of the metal.

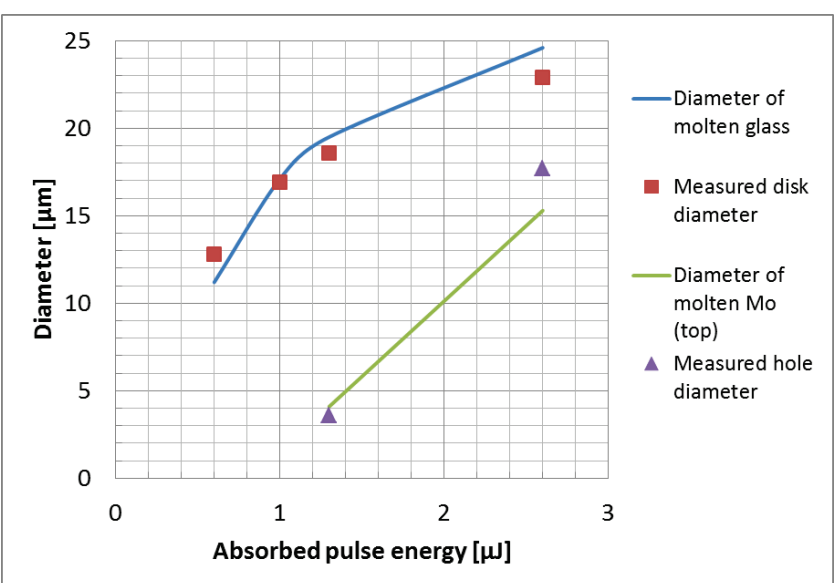

Fig. 9: Comparison of the results of a transient thermal finite element model of the P1 scribe with experimental results [18]. The laser beam diameter is $25 \mu \mathrm{m}$.

\subsection{Simulation of the $P 3$ scribe}

In the next step we simulated the thermal behavior for the P3 scribe. The distribution of the absorbed power derived from the respective optical model was transferred to a transient thermal finite-element model.

Material parameters were taken from the literature $[1,6$, 11]. In contrast to the simulation of the $P 1$ scribe the availability of parameters for typical materials of thin-film solar cells is relatively poor. In particular, there are no parameters available at elevated temperatures. Thus we had to extrapolate the values from the situation at room temperature. That's why we expect the simulation of $\mathrm{P} 3$ ablation to give only qualitative agreement. 
The laser parameters correspond to an excitation at $515 \mathrm{~nm}$ (compare to fig. 8) with an incident pulse energy of $1.1 \mu \mathrm{J}$. The total absorbed energy amounts $0.61 \mu \mathrm{J}$, where $0.43 \mu \mathrm{J}$ are deposited in the top $50 \mathrm{~nm}$ of CIGS, $0.12 \mu \mathrm{J}$ in the CdS buffer, and $0.06 \mu \mathrm{J}$ in the TCO layer.

The finite-element simulation yields a shallow temperature distribution (see fig. 10), which reaches its maximum temperature approximately $70 \mathrm{~nm}$ below the interface between CIGS and the CdS buffer layer. The respective ablation process removes the front (TCO-) electrode only and leaves the CIGS layer intact. Experimental results [19] show an ablation diameter of $20 \mu \mathrm{m}$ for a pulse energy of $1.1 \mu \mathrm{J}$, while the simulation yields a diameter of molten CIGS of $16 \mu \mathrm{m}$ after $10 \mathrm{~ns}$.

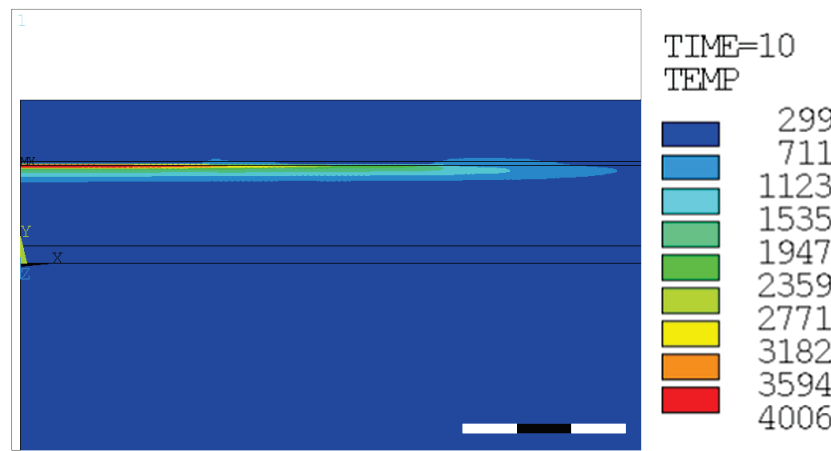

Fig. 10: Temperature distribution $[\mathrm{K}]$ at $10 \mathrm{~ns}$ after the laser pulse. The values of absorbed power are given in the text. The laser beam diameter is $28 \mu \mathrm{m}$. The bar on the bottom of the image is $3 \mu \mathrm{m}$ long.

The finite-element simulation yields a shallow temperature distribution (see fig. 10), which reaches its maximum temperature approximately $70 \mathrm{~nm}$ below the interface between CIGS and the CdS buffer layer.

Experimental results [19] show an ablation diameter of $20 \mu \mathrm{m}$ for a pulse energy of $1.1 \mu \mathrm{J}$. The respective ablation process removes the front (TCO-) electrode only and leaves the CIGS layer intact.

The simulation yields a diameter of molten CIGS of $16 \mu \mathrm{m}$ after $10 \mathrm{~ns}$. This is consistent with delamination in the uppermost part of the CIGS or at the interface between CIGS and the CdS buffer layer as the relevant mechanism of the ablation process.

The scaling of the simulated diameter of molten CIGS with the pulse energy is depicted in fig. 11 for different delay times after the laser pulse. The experimental result compares best with a delay of $5 \mathrm{~ns}$, though the actual time when delamination occurred in the experiment is not known.

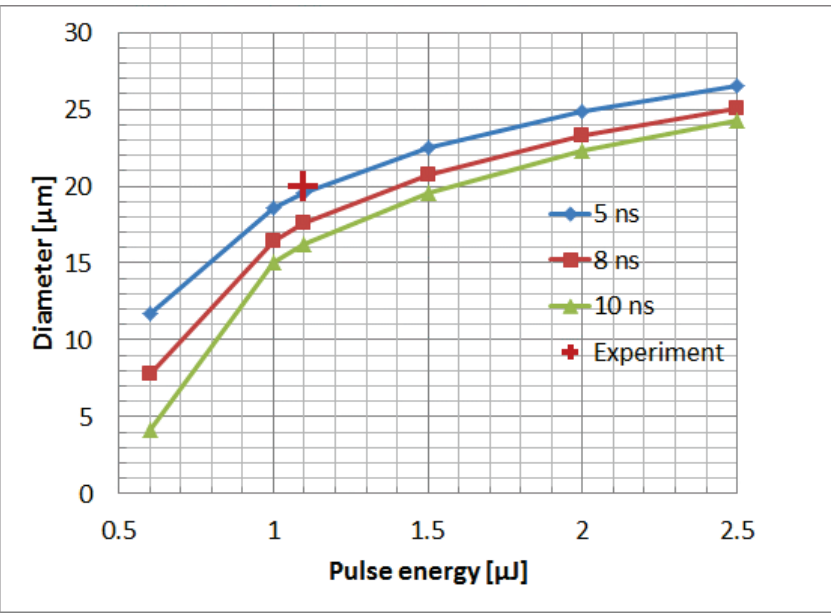

Fig. 11: Simulated diameter of molten CIGS as a function of the pulse energy. The time delay after the laser pulse is used as a parameter. The beam diameter is $28 \mu \mathrm{m}$.

\section{Conclusions}

We presented an optical and thermal model of the structuring of thin-film solar cells by laser ablation. The model takes into account effects of the carrier dynamics in the respective materials which typically leads to a vertical shift of the absorbing region, as well as to changes in the incoupling efficiency for the laser light.

Carrier-induced shielding makes it difficult to find an appropriate process window for P3 structuring to remove the whole stack in a confined ablation process. When using picosecond pulses it is preferable to remove the front electrode only.

Similar shielding effects will occur for P2 structuring with short pulses.

The simulations yield good agreement with current P1 ablation experiments $[17,18]$, and reasonable agreement with the experiments on P3 structuring [19].

\section{Acknowledgments}

This work was supported by BMBF/VDI (Germany) as a part of the T4nPV project under contract no. $13 \mathrm{~N} 11788$.

We acknowledge the good cooperation and many fruitful discussions within the T4nPV project, in particular with Dominik Bartl, Andreas Letsch, and Mawuli Ametowobla from Robert-Bosch $\mathrm{GmbH}$.

\section{References}

[1] M. Bleicher: "Halbleiter-Optoelektronik", (Hüthig, Heidelberg, 1986).

[2] G. Eberhardt, H. Banse, U. Wagner, and T. Peschel: Proc. SPIE 7585, Laser-based Micro- and Nanopackaging and Assembly IV, (2010) 75850P.

[3] H.P. Huber, M. Domke, S. Rapp, J. Sotrop, R. Moser: Proc. SPIE 8972, Frontiers in Ultrafast Optics: Biomedical, Scientific, and Industrial Applications XIV, San Francisco, (2014) p. 897238.

[4] J. Sotrop, A. Kersch, M. Domke, G. Heise, and H.P. Huber: Appl. Phys. A, 113, (2013) 397.

[5] S. Grubinskas, G. Raciukaitis: Proc. 2011 COMSOL Conference, Stuttgart, (2011).

[6] A.D. Compaan, I. Matulionis, and S. Nakade: "Optimization of Laser Scribing for Thin-Film PV Mod- 
ules", (National Renewable Energy Laboratory, Golden, 1998).

[7] K. Orgassa, U. Rau, H.-W. Schock, and J.H. Werner: Proc. of 3rd World Conference on Photovoltaic Energy Conversion, Osaka, (2003) p. 372.

[8] S. Theodoropoulou, D. Papadimitriou, K. Anestou, Ch. Cobet, and N. Esser: Semicond. Sci. Technol. 24, (2009) 015014.

[9] A.M. Hermann et al.: Solar Energy Materials \& Solar Cells, 70, (2001) 345

[10] B. L. Evans and R. A. Hazelwood: phys. stat. sol. (a), 4, (1971) 181.

[11]C. Klingshirn: ChemPhysChem, 8, (2007) 783.

[12]E. D. Palik: "Handbook of Optical Constants of Solids", (Academic, New York, 1985).

[13] M. Born, E. Wolf: "Principles of Optics", 7th ed., (Cambridge Univ. Press, Cambridge, 1999).

[14]B. Rethfeld: Phys. Rev. B, 73, (2006) 035101.
[15]A. Sommerfeld, H. Bethe: "Handbuch der Physik" Vol. 24- 2, (Heidelberg, 1933).

[16] M. Gloeckler, Proc. of 3rd World Conference on Photovoltaic Energy Conversion, Osaka, (2003) p. 491.

[17] D. Bartl, M. Ametowobla, F. Schmid, A. Letsch, M. Hafner, S. Nolte, A. Tünnermann: Optics Express, 21, (2013) 16431.

[18]D. Bartl, A. Michalowski, M. Hafner, A. Letsch, S. Nolte, A. Tünnermann: Appl. Phys. A, 110, (2013) 227.

[19] T. Peschel, G. Matthäus, S. Nolte, R. Eberhardt, A. Tünnermann: Proc. SPIE 8967, Laser Applications in Microelectronic and Optoelectronic Manufacturing (LAMOM) XIX, San Francisco, (2014) p. 896714.

[20] ANSYS Inc.: "ANSYS Mechanical APDL Theory Reference", Release 15.0, (ANSYS Inc., Canonsburg, 2013).

(Received: June 17, 2014, Accepted: August 17, 2015) 